\title{
Risk Analysis of Volume Cheat Strategy in a Competitive Capacity Market
}

\author{
Feng, Donghan; Xu, Zhao
}

Published in:

IEEE Power \& Energy Society General Meeting, 2009. PES '09.

Link to article, DOI:

10.1109/PES.2009.5275804

Publication date:

2009

Document Version

Publisher's PDF, also known as Version of record

Link back to DTU Orbit

Citation (APA):

Feng, D., \& Xu, Z. (2009). Risk Analysis of Volume Cheat Strategy in a Competitive Capacity Market. In IEEE Power \& Energy Society General Meeting, 2009. PES '09. (pp. 1-7). IEEE.

https://doi.org/10.1109/PES.2009.5275804

\section{General rights}

Copyright and moral rights for the publications made accessible in the public portal are retained by the authors and/or other copyright owners and it is a condition of accessing publications that users recognise and abide by the legal requirements associated with these rights.

- Users may download and print one copy of any publication from the public portal for the purpose of private study or research.

- You may not further distribute the material or use it for any profit-making activity or commercial gain

- You may freely distribute the URL identifying the publication in the public portal 


\title{
Risk Analysis of Volume Cheat Strategy in a Competitive Capacity Market
}

\author{
Donghan Feng, Zhao Xu Member IEEE
}

\begin{abstract}
Capacity market provides additional revenue stream for the power suppliers. In a capacity-energy combined market environment, suppliers have incentives to deliberately over-offer their capacities in the capacity market while bid very high price in the energy and ancillary markets to avoid operation. This paper analyzes the risks and profits of this capacity-overoffer behavior, and develops a method for computing nonoperable penalty level which can prevent the capacity-over-offer behavior. It is found that the effective penalty level is highly correlated with the stochastic characteristics of the supplier's profit streams and attitudes towards risk. Two types of suppliers are identified with high potential of capacity cheating behavior in the analysis. The methodology and the results are potentially useful for regulating participants' misbehaviors and enhancing the operation security in a capacity-energy market environment.

Index Terms: capacity market; volume cheat; risk management; Prospect Theory; Monte-Carlo simulation.
\end{abstract}

\section{INTRODUCTION}

Capacity market is one approach to address the long-term generation resource adequacy problem. In northeast US, capacity markets have been in operation for almost ten years. Capacity market is an explicit mechanism for pricing resource reliability, which yields an explicit/separate price signal for generation investment. A capacity market also provides generators with additional revenue stream besides energy/ancillary markets. These revenues are important for peaking generators which have "missing money" problem [1][2][3]. The disadvantage of capacity market approach is its administrative essence. Some argue that creating capacity markets will delay the development of a sufficient demand response, which is the right way to ultimately address resource adequacy problem. They believe in other approaches, such as forward contracts and call options to ensure generation investment [4][5][6][7].

In a capacity-energy combined market environment, the strategies of power suppliers will be different from those in the energy-only market environment, due to the change of their money streams.

In this paper, we focus on issues in capacity-energy combined market environment. The capacity requirement is calculated by the forecasted peak load plus a certain margin, therefore, the generation capacities cleared in the capacity

D. Feng is with the Department of Electrical Engineering of Shanghai Jiao Tong University, Shanghai, China and will be also with Centre for Electric Technology, Technical University of Denmark from 2009 (e-mail: seed@sjtu.edu.cn).

Z. $\mathrm{Xu}$ is with Centre for Electric Technology, Technical University of Denmark, DK-2800 Lyngby, Denmark (e-mail: zx@elektro.dtu.dk) market is always higher than the real peak load. Then, a lot of generation capacities will not operate indeed. Therefore, suppliers may cheatingly offer more capacity than they actually have, and bid high price in the energy market to avoid dispatch. This strategy can bring generators additional revenue without costs, but may cause serious operation problems for the system operator.

From a supplier's viewpoint, over-offering can bring additional revenue stream, but on the other hand, this strategy may also incur penalty when disclosed as non-operable in reality. The real peak load during the capacity period can be much higher than predicted. Moreover, other circumstances such as the outage of a large generator, the emergency start need from a local blackout, may also require unexpected activation of the cheating capacity, no matter how high their bidding price in the energy/reserve market is. When called for operation and revealed as non-operable, the cheating supplier will suffer the penalty.

Therefore, whether the strategy of over-offering is profitable depends on a number of factors, including the nonoperable penalty level, the load forecast accuracy, the probability of potential operation, the capacity market price, the system capacity adequacy requirement and the risk attitudes of the suppliers.

This paper analyzes the potential return and associated risk of the over-offering strategy, as well as their relationship with the above factors. The motivation is to find a penalty mechanism that can make this strategy less profitable and more risky for the potential cheaters to exercise.

The remaining sections are organized as follows. Section II first analyzes the risks and profits of this capacity-over-offer behavior, and then develops a method of computing nonoperable penalty level which can prevent the capacity-overoffer behavior of suppliers with different risk attitudes. In Section III, simulation results of three different types of suppliers are presented and discussed, it is found that riskneutral penalty level can be either too high or too low for suppliers with different cash streams. In Section IV, some conclusions are drawn.

\section{Methodology}

In this section, the profits and risks of the capacity-overoffer behavior are analyzed and the methods for setting nonoperable penalty level under different risk attitudes are developed. This section consists of three subsections, Subsection A focuses on formulating the random characteristics of the money stream of the capacity-over-offer 
behavior. Subsection B deduces the analytical form of a penalty level which can prevent capacity-over-offer behavior for risk-neutral participants. Subsection C develops a penalty setting algorithm for risk-averse and risk-loving participants.

\section{A. Formulating effective penalty mechanism}

Assume a supplier offer $\mathrm{x}(\mathrm{MW})$ cheating capacity in the capacity market. It will receive $\tilde{p}_{C} \cdot x$ extra revenue, where $\tilde{p}_{C}$ denotes the capacity price. Since the supplier should decide $\mathrm{x}$ before the capacity market clears, $\tilde{p}_{C}$ has uncertainty, so an upper-swung-dash is used to express that capacity price is a random variable.

Therefore the profit of over-offering can be formulated as:

$$
\tilde{\pi}_{c}=\tilde{p}_{C} \cdot x-\tilde{B}
$$

where $\tilde{B}$ denotes the total penalty (\$) the cheater will suffer. The term $\tilde{B}$ is related with the amount of cheating capacity which will be exposed, here we use $\tilde{y}(x, t)$ to denote the exposed capacity in operation interval $t$ when offering $x$ cheating capacity. Then we have:

$$
\tilde{B}=\sum_{t=1}^{T O I} \mathrm{M}(\tilde{y}(x, t))
$$

where $\mathrm{M}(\bullet)$ denotes the penalty mechanism.

The next step is to formulate $\tilde{y}(x, t)$. The formulation of $\tilde{y}(x, t)$ may differ significantly given different market designs and operation rules. Usually, the exposed capacity $\tilde{y}(x, t)$ is correlated with scarcity/shortage pricing mechanisms in operation. For adequacy and security concern, electricity markets/power systems normally have a certain form of scarcity/shortage pricing mechanisms, which will be effective in tight supply-demand conditions. The scarcity/shortage pricing mechanisms usually require all available system capacities to be activated and compensated at a predetermined price (often very high, close to the VOLL value) if called for operation. Scarcity pricing programs in different electricity markets are triggered by different conditions. Here we simply assume that the scarcity pricing program is triggered by the demand level, when demand exceeds a threshold level $\bar{D}$, all the capacities in the system will be under central operation and receive an administrative price $p_{S C}$ for each MW generation.

Under this scarcity pricing program, $\tilde{y}(x, t)$ can be classified into three situations. When demand is less than the scarcity threshold, or $\tilde{D}(t) \leq \bar{D}$, cheating capacity has no risk to be exposed, or $\tilde{y}(x, t)=0$; when demand is higher than the total system capacity, or $\tilde{D}(t) \geq C$, all cheating capacity will be called for operation, or $\tilde{y}(t)=x$; when demand lies between scarcity threshold and system capacity, or $\bar{D}<\tilde{D}(t)<C$, certain amount of cheating capacity has certain probability of exposure, or $\tilde{y}(t)$ is a random variable following the hypergeometric distribution.

Therefore, $\tilde{y}(x, t)$ can be formulated as:

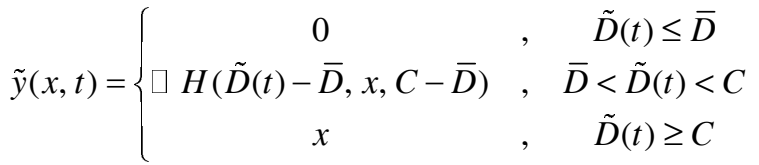

where $H(\alpha, \beta, \tau)$ denotes the hypergeometric distribution function with the parameters $\alpha, \beta$ and $\tau$. Here $\alpha=\tilde{D}(t)-\bar{D}, \beta=x, \quad \tau=C-\tilde{D}(t)$. Intuitively, this means choosing $\tilde{D}(t)-\bar{D}$ from all the available capacity $C-\bar{D}$, in which $x$ is cheating.

The hypergeometric distribution implies that the possibility of disclosing y MW cheating capacity follows:

$$
\begin{aligned}
& \mathrm{P}(\tilde{y}(x, t)=y)=\frac{C_{x}^{y} \cdot C_{C-\tilde{D}(t)-x}^{\tilde{D}(t)-\bar{D}}}{C_{C-\tilde{D}(t)}^{\tilde{D}(t)}} \\
& =\frac{x !(C-\tilde{D}(t)-x) !(C-2 \cdot \tilde{D}(t)+\bar{D}) !(\tilde{D}(t)-\bar{D}) !}{(x-y) ! y !(C-2 \tilde{D}(t)+\bar{D}-x+y) !(\tilde{D}(t)-\bar{D}-y) !(C-\tilde{D}(t)) !}
\end{aligned}
$$

Substitute (3) and (2) into (1), we can get the analytical formulation of the cheating profit:

$$
\tilde{\pi}_{c}=\tilde{p}_{C} \cdot x-\sum_{t=1}^{T O I} \mathrm{M}(\tilde{y}(x, t))
$$

where $\tilde{y}(x, t)$ follows (3).

The classical method to compare the preference of random money stream is the Expected Utility Theory (EUT) [9]. It is assumed in EUT that an investor's objective is to maximize the expected utility, i.e., the expected value of his utility function. Based on EUT, the problem of over-offer prevention can be expressed as:

Finding an optimal mechanism $\mathrm{M}^{*}(\bullet)$, to ensure $\forall x>0$,

$$
\mathrm{E}\left[\mathrm{U}\left(W_{0, i}+\tilde{\pi}_{n, i}+\tilde{\pi}_{c}\right)\right]<\mathrm{E}\left[\mathrm{U}\left(W_{0, i}+\tilde{\pi}_{n, i}\right)\right]
$$

To show the penalty mechanism explicitly, (6) can be rewritten as:

$$
\mathrm{E}\left[\mathrm{U}\left(W_{0, i}+\tilde{\pi}_{n, i}+\tilde{p}_{C} \cdot x-\sum_{i=1}^{T O I} \mathrm{M}(\tilde{y})\right)\right]<\mathrm{E}\left[\mathrm{U}\left(W_{0, i}+\tilde{\pi}_{n, i}\right)\right]
$$

Inequality (7) is the criteria for an effective penalty mechanism. Here $\mathrm{E}[\bullet]$ denotes the mathematical expectation, $\mathrm{U}(\bullet)$ denotes the utility function, $W_{0, i}$ is the wealth level of supplier $i$ when making the decision (or initial endowment), generally, $W_{0, i}$ can be formulated as:

$W_{0, i}=A_{i}-L_{i}$

where $A_{i}$ denotes the present value of total assets, $L_{i}$ denotes the present value of total liabilities.

$\tilde{\pi}_{n, i}$ denotes the normal profit of supplier $i$. The normal profit is a random variable based on the prices of energy and ancillary services. For example, the normal profit of a thermal generator $j$ participating in capacity market, spot energy market, fixed contract and reactive power may read as:

$$
\begin{aligned}
& \tilde{\pi}_{n, j}=\tilde{p}_{C} \cdot C_{j}+p_{F C} \cdot P_{F C}+\tilde{p}_{S P} \cdot P_{S P}+p_{Q} \cdot Q_{j}-\mathrm{f}_{c, j}\left(P_{F C}+P_{S P}\right)(9) \\
& \text { s.t. }
\end{aligned}
$$

where $P_{F C}$ and $P_{S P}$ denotes the generation in fixed contract 
and spot market, $Q_{j}$ denotes the supply of reactive power, $C_{j}$ denotes the capacity of the generator, $V_{t}$ denotes the voltage at the generator terminal bus, $I_{a}$ denotes the steadystate armature current, $\mathrm{f}_{c, j}(\bullet)$ denotes the cost function, the most widely used forms are linear and quadratic cost functions.

Equations (9)-(11) demonstrate a simple example of modeling suppliers' normal profit and associated constraints. A more complicated model can include incomes from other ancillary services and more deliberately multi-trading strategies [10].

\section{B. Deducing Risk Neutral Secure Penalty}

The solution of the problem of preventing over-offer, or optimal penalty $\mathrm{M}^{*}(\bullet)$ could have various forms. Within them, the commonly applied mechanism is to penalize each unit of inoperable capacity by a fixed penalty $b$, or, $\mathrm{M}(\tilde{y})=b \cdot \tilde{y}$, then (7) can be written as:

$\mathrm{E}\left[\mathrm{U}\left(W_{0, i}+\tilde{\pi}_{n, i}+\tilde{p}_{C} \cdot x-b \cdot \sum_{t=1}^{T O I} \mathrm{E}[\tilde{y}]\right]<\mathrm{E}\left[\mathrm{U}\left(W_{0, i}+\tilde{\pi}_{n, i}\right)\right]\right.$

Generally, the lowest secure penalty level can be expressed as:

$\underline{b}=\inf \left\{\begin{array}{l}b \in \square, \forall x>0: \\ W_{0}+\mathrm{E}\left(\tilde{\pi}_{n, i}\right)+\mathrm{E}\left(\tilde{p}_{C}\right) \cdot x-b \cdot \sum_{t=1}^{T O I} \mathrm{E}(\tilde{y}) \leq W_{0}+\mathrm{E}\left(\tilde{\pi}_{n, i}\right)\end{array}\right\}$

(13)

where inf $\{\bullet\}$ denotes the inferior limit.

For risk-neutral suppliers, we can have a more attractive form of $\underline{b}$. Notice that $\mathrm{U}(\bullet)$ is monotonically increasing, and for a risk-neutral supplier, $\mathrm{E}[\mathrm{U}(\tilde{W})]=\mathrm{U}(\mathrm{E}[\tilde{W}])$. Therefore, condition (12) can be rewritten as:

$W_{0}+\mathrm{E}\left[\tilde{\pi}_{n, i}\right]+\mathrm{E}\left[\tilde{p}_{C}\right] \cdot x-b \cdot \sum_{t=1}^{T O I} \mathrm{E}[\tilde{y}]<W_{0}+\mathrm{E}\left[\tilde{\pi}_{n, i}\right]$

Or equivalently:

$\mathrm{E}\left[\tilde{p}_{C}\right] \cdot x-b \cdot \sum_{t=1}^{T O I} \mathrm{E}[\tilde{y}]<0$

Notice that:

$\mathrm{E}[\tilde{y}]$

$=\operatorname{Pr}(\tilde{D}(t) \leq \bar{D}) \cdot 0+\operatorname{Pr}(\bar{D}<\tilde{D}(t)<C) \cdot x \cdot \frac{\tilde{D}(t)-\bar{D}}{C-\tilde{D}(t)}+x \cdot \operatorname{Pr}(\tilde{D}(t) \geq C)$

$=\operatorname{Pr}(\bar{D}<\tilde{D}(t)<C) \cdot x \cdot \frac{\tilde{D}(t)-\bar{D}}{C-\tilde{D}(t)}+x \cdot \operatorname{Pr}(\tilde{D}(t) \geq C)$

$=\left\{\operatorname{Pr}(\bar{D}<\tilde{D}(t)<C) \cdot \frac{\tilde{D}(t)-\bar{D}}{C-\tilde{D}(t)}+\operatorname{Pr}(\tilde{D}(t) \geq C)\right\} \cdot x$

(16)

Substitute (16) into (15), we have:

$\left\{\mathrm{E}\left[\tilde{p}_{C}\right]-b \cdot \sum_{t=1}^{T O I}\left\{\operatorname{Pr}(\bar{D}<\tilde{D}(t)<C) \cdot \frac{\tilde{D}(t)-\bar{D}}{C-\tilde{D}(t)}+\operatorname{Pr}(\tilde{D}(t) \geq C)\right\}\right\} \cdot x<0$

(17)

since $x>0,(17)$ is equivalent to:
$\mathrm{E}\left[\tilde{p}_{C}\right]-b \cdot \sum_{t=1}^{T O I}\left\{\operatorname{Pr}(\bar{D}<\tilde{D}(t)<C) \cdot \frac{\tilde{D}(t)-\bar{D}}{C-\tilde{D}(t)}+\operatorname{Pr}(\tilde{D}(t) \geq C)\right\}<0$

or:

$b>\mathrm{E}\left[\tilde{p}_{C}\right] / \sum_{t=1}^{T O I}\left\{\operatorname{Pr}(\bar{D}<\tilde{D}(t)<C) \cdot \frac{\tilde{D}(t)-\bar{D}}{C-\tilde{D}(t)}+\operatorname{Pr}(\tilde{D}(t) \geq C)\right\}$

(18)

Hence, the lower limit of non-operable penalty level which can prevent risk-neutral participants' capacity-over-offer behavior has been obtained. In this paper, $\underline{b}_{R N}$ is used to denote this level, then we have:

$\underline{b}_{R N}=\mathrm{E}\left(\tilde{p}_{C}\right) / \sum_{t=1}^{T O I}\left\{\operatorname{Pr}(\bar{D}<\tilde{D}(t)<C) \cdot \frac{\tilde{D}(t)-\bar{D}}{C-\tilde{D}(t)}+\operatorname{Pr}(\tilde{D}(t) \geq C)\right\}$ (19)

A penalty higher than $\underline{b}_{R N}$ can ensure that any risk-neutral supplier suffers a loss when bid a non-zero cheating capacity in the capacity market. Therefore, a rational risk-neutral supplier will not cheat in the capacity market under $\underline{b}_{R N}$ penalty. In this aspect, we call $\underline{b}_{R N}$ the risk-neutral-secure penalty (RNS penalty).

We can notice that $\underline{b}_{R N}$ depends only on the expectation of capacity market price $\mathrm{E}\left[\tilde{p}_{C}\right]$, demand level $\tilde{D}(t)$, scarcity threshold $\bar{D}$ and system capacity level $C$, but NOT relates to $\tilde{\pi}_{n, i}$ or $C_{i}$ or any other individual parameters of supplier $i$. In other words, one control area requires only one uniform RNS penalty to prevent cheating behavior, rather than requires different penalty levels for different suppliers.

\section{Analysis for more diverse risk-attitudes}

In the above subsection, the minimal penalty level of a riskneutral supplier is deduced. This RNS penalty can ensure the rational risk-neutral supplier to behave honesty in the capacity market.

However, the risk-neutral assumption is too strong for all suppliers at all times. Risk-neutrality equivalently means that all suppliers concern only about their expected profit no matter what the risk is. This is not always the situation, some suppliers do concern about their risks. The more general case is that suppliers concern about their expected profit as well as the associated risk. Therefore, this RNS penalty may be too high or too low for risk-averse and risk-loving suppliers.

The most widely accepted theorem concerning risk attitudes is the Prospect Theory ${ }^{1}$. Prospect Theory by experimental methodology discovered that decision-makers are risk-averse for gains and risk-loving for losses. Readers can refer to [8] for more details about Prospect Theory.

In electricity markets, most suppliers are making money, so they perform risk-averse in decision-making. Base-load/ intermediate suppliers will not offer cheating capacity under the RNS penalty, because cheating capacity will include an extra volatility to their stable normal revenue stream. Under

\footnotetext{
${ }^{1}$ Prospect Theory is established by American economist Daniel Kahneman and Amos Tversky. The winning of 2002 Nobel Prize was regarded as a milestone of the worldwide acknowledgement of Prospect Theory.
} 
the RNS penalty, the cheating behavior is highly risky. If seldom called for operation, the cheating behavior will not be exposed and cheating supplier will receive extra pay from the capacity market with no fixed or variable cost. But if frequently called for operation, the cheating capacity can incur huge amount of penalty. This "gambling" behavior is hence not preferred by risk-averse base-load suppliers.

There are two types of potential cheaters. The first type is the profit-losing base-load/intermediate suppliers. They are risk-loving and inclined to take a more risky strategy such as the cheating behavior.

The second type is profit-making peaking-load suppliers (peakers), because their normal profit is negatively correlated with their cheating profit. When the real demand is higher than expected, peakers will generate more and gain more normal profit; meanwhile their cheating profit will also be lower than expected, because the probability of disclosure of their cheating bidding will be higher than expected due to the high demand. When the real demand is lower than expected, peakers will generate less and gain less normal profit, meanwhile the probability of the potential penalty is also less, resulting in more cheating profit. In this manner, peakers' normal profit is negatively correlated with their cheating profit and the total profit (the cheating plus normal profit) will be more stable. This stable revenue stream is preferable for risk-averse suppliers, even though the expected profit is theoretically the same under RNS penalty. Therefore, peakers will more probably (than baseload suppliers) offer a certain amount of cheating capacity in the capacity market, to stabilize their money stream.

The general model for extracting a secure penalty level under various risk attitudes is formulated as the following:

$\min _{b} \max _{i} b$

$$
\text { s.t. } \forall 0 \leq x \leq \bar{x}, \mathrm{E}\left[\mathrm{U}\left(W_{0, i}+\tilde{\pi}_{n, i}+\tilde{p}_{C} \cdot x-b \cdot \sum_{i=1}^{T O I} \tilde{y}\right)\right]<\mathrm{E}\left[\mathrm{U}\left(W_{0}+\tilde{\pi}_{n, i}\right)\right]
$$

The risk-averse/ risk-loving degree is implied in the concavity/convexity of the utility function $\mathrm{U}(\bullet)$. If the utility function is concave, it embodies risk-averse. If the utility function is convex, it embodies risk-loving.

Here, since $\mathrm{U}(\bullet)$ is nonlinear, $\mathrm{E}[\mathrm{U}(\tilde{W})] \neq \mathrm{U}(\mathrm{E}[\tilde{W}])$, the random variables $\tilde{S}_{0}, \quad \tilde{p}_{C}$ and $\tilde{y}(t) \mathrm{s}$ can not be easily decoupled and it is impossible to derive an analytical form of secure-penalty.

However, (20)-(21) can still be solved through numerical algorithms. In this paper, we use a Monte-Carlo simulation to obtain numerical solutions of secure penalty under general risk attitudes. The Flow Chart of the proposed algorithm is illustrated in Fig. 1.

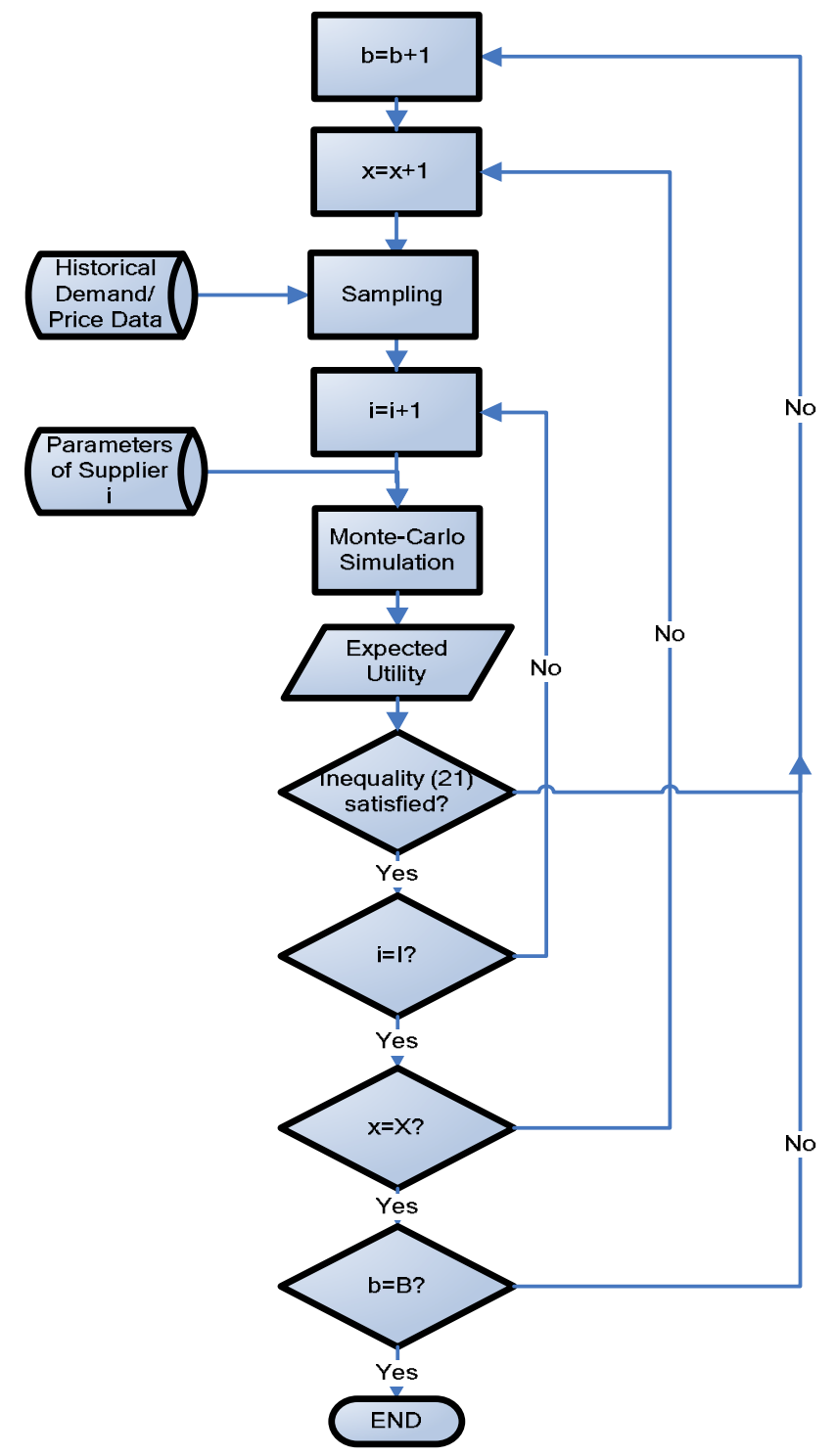

Fig. 1. Flow Chart of the Proposed Algorithm (I: number of suppliers; x: upper limit of cheating capacity; B: upper limit of penalty level)

\section{Testing Results}

In the previous section, the problem of participant's cheating behavior is formulated, the analytical form of secure penalty under risk-neutral assumption is deduced and a simulation algorithm for calculating secure penalty under general risk attitudes is developed.

The previous section also pointed out two potential cheaters. In this section, the proposed approaches for calculating a secure penalty will be tested based on real market data. The expected value and volatility of different suppliers' cheating and normal profit will be compared.

Assume there are three GenCos in the market. GenCoA owns two 100MW gas-fueled peaking generators. Due to the high gas price, his strategy is to generate only in the scarcity intervals.

GenCoB owns a 300MW coal-fired generator. Due to its very low fuel cost and high startup cost, its strategy is to 
generate full capacity as continuously as possible. So it has signed fixed price contract with full capacity.

GenCoC owns a 100MW off-shore wind farm. It has no fuel cost and little operation cost. Most of its cost comes from the annualized depreciation charge. But the rise of the cost of anti-corrosion coatings caused by the soaring crude oil price results in the unexpected high maintenance cost.

In this section, the cost of the GenCos are divided as three parts, depreciation charge $c_{D}$, which is assumed fixed and calculated as $\$ / \mathrm{MWy}$, Operation and Maintenance cost $c_{O M}$ which is assumed fixed and calculated as $\$ / \mathrm{MWy}$, and the fuel cost $c_{F}$, which depends on the output (MW) and calculated as \$/MWh.

Based on the above setup, the normal profit of the three GenCos are formulated as:

$$
\begin{aligned}
& \tilde{\pi}_{n, A}=\tilde{p}_{C} \cdot C_{A}-c_{D, A}-c_{O M, A}+\sum_{t=1}^{T O I}\left(p_{s}-c_{F, B}\right) \cdot \tilde{z}(t) \\
& \tilde{\pi}_{n, B}=\tilde{p}_{C} \cdot C_{B}-c_{D, B}-c_{O M, B}+\left(p_{F C, B}-c_{F, B}\right) \cdot C_{B} \cdot T_{O, B} \\
& \tilde{\pi}_{n, C}=\tilde{p}_{C} \cdot C_{C}-c_{D, C}-c_{O M, C}+\sum_{t=1}^{T O I}\left(p_{S P}(t)-c_{F, C}\right) \cdot P(t)
\end{aligned}
$$

where

$$
\tilde{z}(t)=\left\{\begin{array}{ccc}
0 & , & \tilde{D}(t) \leq \bar{D} \\
\tilde{z}(t) \square H\left(\tilde{D}(t)-\bar{D}, C_{i}, C-\tilde{D}(t)\right) & , & \bar{D}<\tilde{D}(t)<C \\
C_{i} & , & \tilde{D}(t) \geq C
\end{array}\right.
$$

For comparison, it is assumed that (1) the present value of assets minus liabilities, or $W_{0, i}$ of the three suppliers are the same; (2) the utility functions of the three suppliers are the same, shown as:

$$
\mathrm{U}(\tilde{W})= \begin{cases}1-e^{-3 \cdot\left(\tilde{W}-W_{0}\right) / W_{0}}, & \tilde{W} \geq W_{0} \\ e^{3 \cdot\left(\tilde{W}-W_{0}\right) / W_{0}}-1, & \tilde{W}<W_{0}\end{cases}
$$

where $\tilde{W}=W_{0}+\tilde{\pi}_{n}+\tilde{\pi}_{c}$. This utility function denotes riskaverse in gain and risk-loving in loss.

In this simulation, the parameters are set as: $W_{0, A}=W_{0, B}=W_{0, C}=3 \times 10^{6} \$ \quad, \quad p_{S C}=1000 \$ / M W h \quad$, $T_{O, B}=7000, C_{A}=200 M W, C_{B}=300 M W, C_{C}=100 M W$, $p_{F C, A}=50 \$ / M W h \quad, \quad c_{D, A}=20000 \$ / M W y$ $c_{D, B}=40000 \$ / M W y \quad, \quad c_{D, C}=60000 \$ / M W y$, $c_{O M, A}=10000 \$ / M W y \quad, \quad c_{O M, B}=20000 \$ / M W y$ $c_{O M, C}=30000 \$ / M W y, c_{F, A}=60 \$ / M W h, c_{F, B}=45 \$ / M W h$ $c_{F, C}=0 \$ / M W h$.

From (19), the risk-neutral secure penalty level can be calculated. The result is $\underline{b}_{R N}=1325$.

However, if $\underline{b}_{R N}$ is used for penalty level, GenCoA and GenCoC will choose the cheating strategy. Fig. 2, Fig. 3 and Fig. 4 show the utilities of normal profits of GenCoA, GenCoB and GenCoC, respectively. We can see that the optimal strategy for GenCoA is to offer $13 \mathrm{MW}$ cheating capacity in the capacity market and for $\mathrm{GenCoC}$ is to offer $30 \mathrm{MW}$ cheating capacity. While for $\mathrm{GenCoB}$, the optimal strategy is not to offer any cheating capacity.

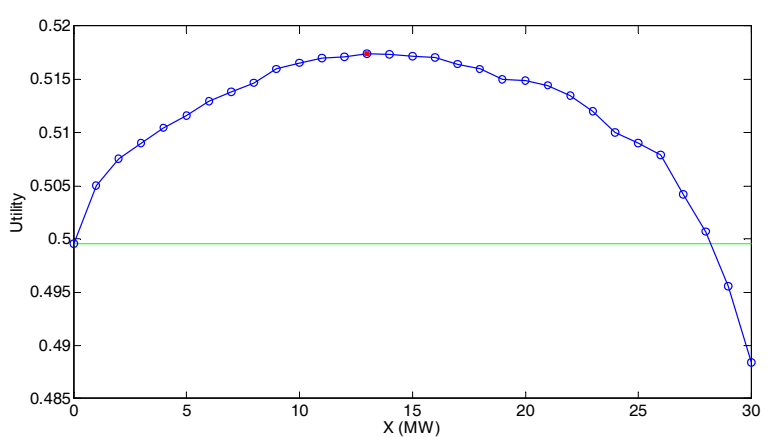

Fig. 2 Utility-x, GenCoA

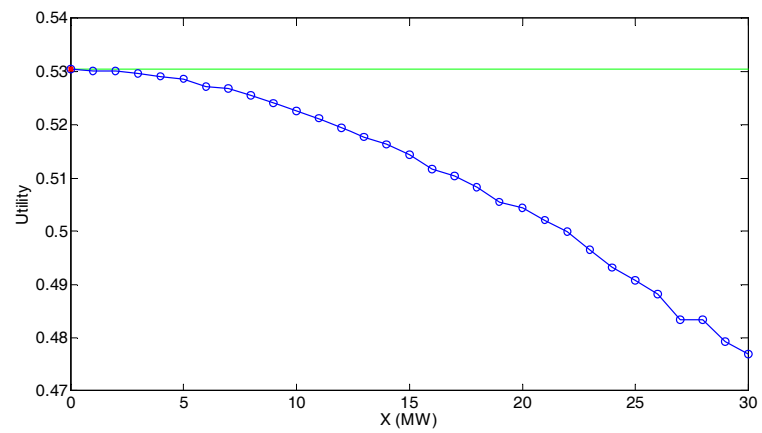

Fig. 3 Utility-x, GenCoB

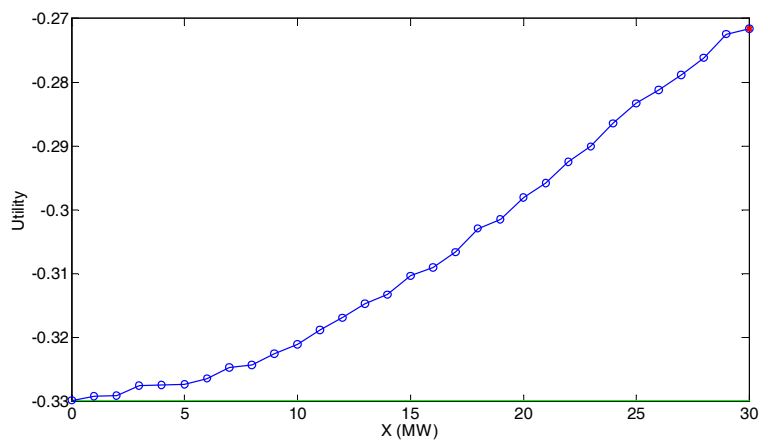

Fig. 4 Utility-x, GenCoC

We can find from (19) that the expected profit is always the same under $\underline{b}_{R N}$ whatever the cheating capacity is. Then why the utility differs significantly under different cheating capacity? The answer exists in the risks. Fig. 5, Fig. 6 and Fig. 7 depict the variance and Value at Risk (VaR) of GenCoA, GenCoB and GenCoC.

$\mathrm{VaR}$ is percentile-based measure, defined as $\mathrm{VaR}_{\mathrm{c}}=\inf \{L \in \square: \operatorname{Prob}(\Delta$ Loss $<L) \leq 1-c\}$, where $\operatorname{Prob}(\bullet)$ denotes conditional probability function, $\Delta$ Loss denotes the potential loss, and $c$ denotes the confidence level (set as $95 \%$ here).

We can see in Fig. 5, Fig. 6 and Fig. 7 that although variance and $\mathrm{VaR}$ are totally different risk measures, they represent almost the same shapes when cheating capacity varies. GenCoA initially can lower its risk by offering more cheating capacity, but after $13 \mathrm{MW}$, its risk will rise with more cheating capacity. The minimal risk point (13MW) is the same with the maximal utility point $(13 \mathrm{MW})$. The risk of 
GenCoB and $\mathrm{GenCoC}$ will always rise by offering more cheating capacity. But while GenCoB's wealth is at the riskaverse section and GenCoC's wealth is at the risk-loving section of the utility function, their optimal cheating capacities are $0 \mathrm{MW}$ and $30 \mathrm{MW}$, respectively.

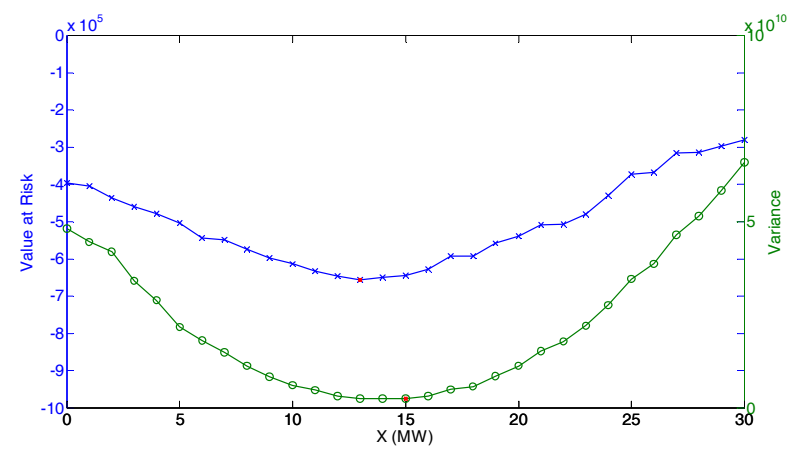

Fig. 5 Risk-x, Peaker

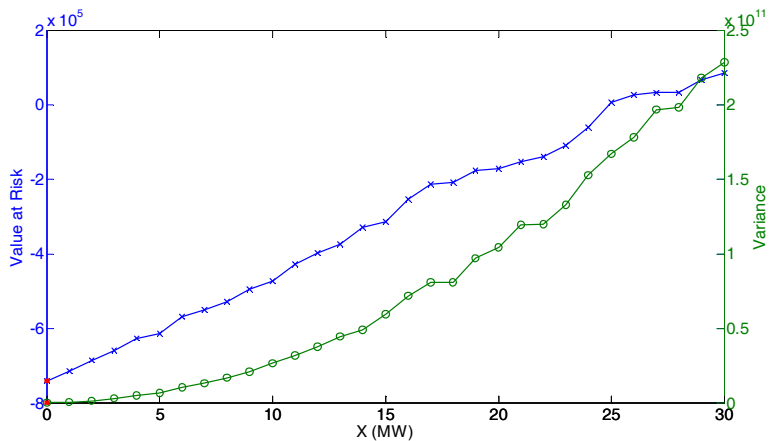

Fig. 6 Risk-x, BaseGen

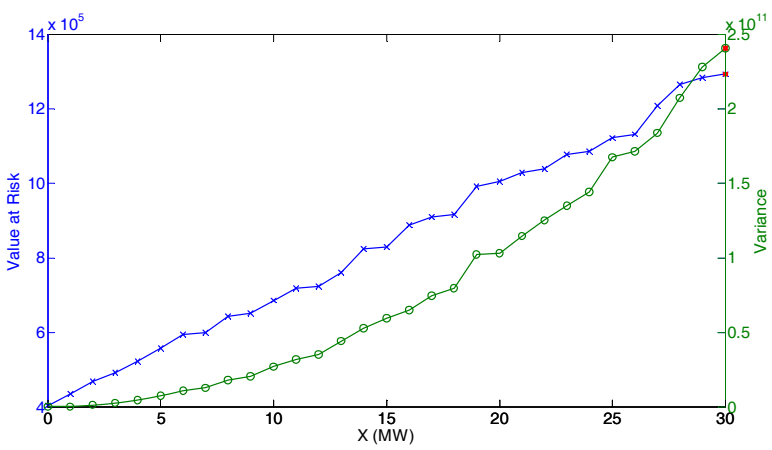

Fig. 7 Risk-x, LosingMoneyGen

The next question is why GenCoA can lower its risk by offering more cheating capacity while GenCoB and GenCoC can not?

To answer this question, we can take a closer look at the probability distribution of their profits before and after cheating, or $\tilde{\pi}_{n}$ and $\tilde{\pi}_{n}+\tilde{\pi}_{c}$. Fig. 8 compare the distributions of pre-cheating (normal) profit and post-cheating (normal+cheating) profit of GenCoA, at the optimal cheating capacity $13 \mathrm{MW}$. We can see that the post-cheating profit is significantly less widely distributed than the pre-cheating profit. In other words, the cheating profit partly hedged the risk in normal profit. The correlation coefficient between normal profit and cheating profit is -0.9819 .

For GenCoB and GenCoC, the correlation between normal profit and cheating profit are all close to zero, 0.0018 and
0.0033 respectively. Fig. 9 and Fig. 10 show the distribution of $\mathrm{GenCoB}$ and $\mathrm{GenCoC}$, we can see that the cheating behavior significantly increases the volatility of their profit stream.

To ensure these three GenCos all abandon the cheating behavior. The algorithm described in Fig. 1 can be used to calculate the penalty level. The result is that penalty level should be lifted to $1482 \$ / M W h$, where $1396 \$$ MWh can ensure GenCoA's non-cheating and 1482\$/MWh can ensure GenCoC's non-cheating.

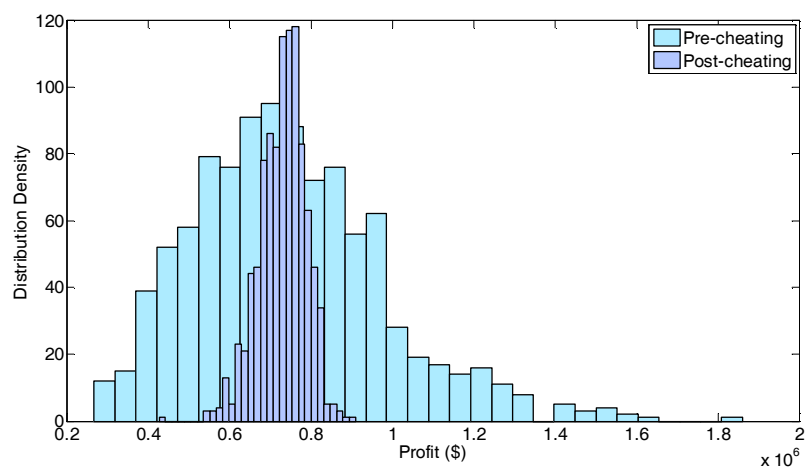

Fig. 8 Comparison between Sample Distribution of GenCoA's Profits before and post cheating

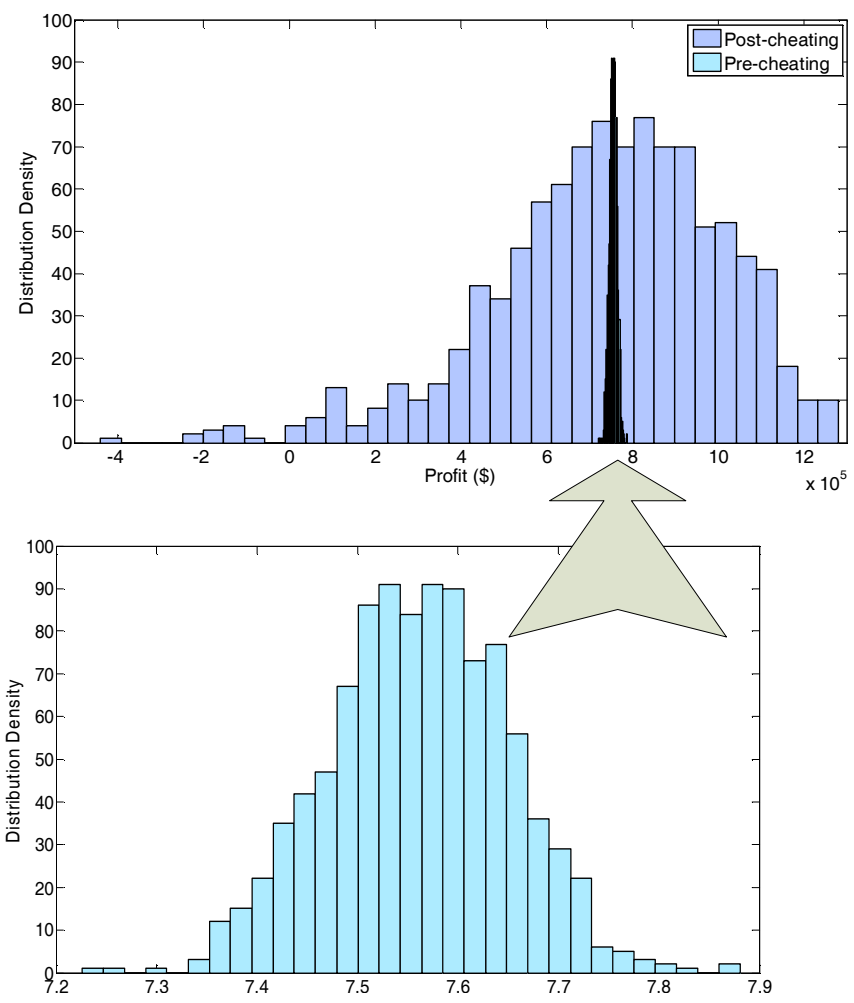

Fig. 9 Comparison between Sample Distribution of GenCoB's Profits before and post cheating 


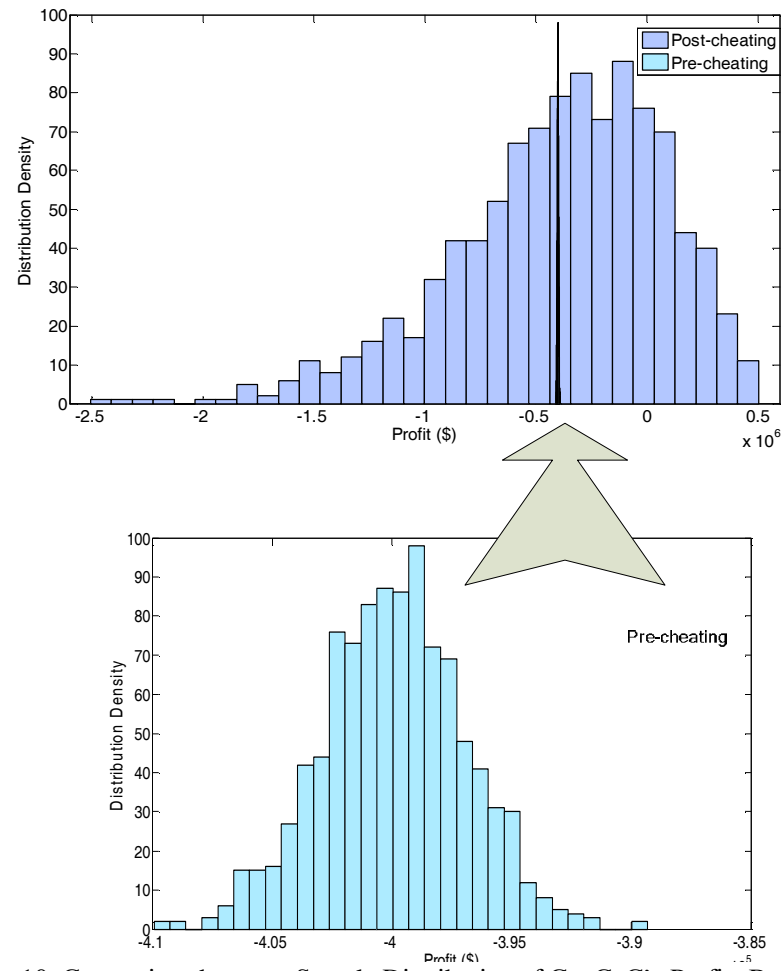

Fig. 10 Comparison between Sample Distribution of GenCoC's Profits Before and Post Cheating

\section{CONCLUSIONS}

In this paper, the strategy of capacity-over-offer is analyzed. For preventing this potentially threatening behavior, the analytical form of risk-neutral non-operable penalty is deduced. An analysis including the correlation between cheating profit and normal profit, as well as the risk attitudes is conducted. A Monte-Carlo simulation embedded computer program was developed for solving the problem. The results suggest that profit-losing base-load/intermediate suppliers and the profit-making peaking-load suppliers still have incentives to over-offer in the capacity market under risk-neutral penalty level. Although the penalty mechanism and the scarcity pricing mechanism adopted in this work are simplified, the methodology suggested is rather general.

\section{REFERENCES}

[1] P. Cramton, S. Stoft, "A Capacity Market that Makes Sense", IEEE Power Engineering Society General Meeting, June 2005, San Francisco, CA, USA.

[2] B.F. Hobbs, M.C. Hu, J.G. Iñón, S.E. Stoft, M.P. Bhavaraju, “A Dynamic Analysis of a Demand Curve-Based Capacity Market Proposal The PJM Reliability Pricing Model", IEEE Trans. Power Systems, vol. 22, no.1, pp. 3-11, January 2007.

[3] J. G. Besser, J. G. Farr and S. F. Tierney, "The political economy of longterm generation adequacy: Why an ICAP mechanism is needed as part of standard market design," Electricity Journal, vol. 15, no. 7, pp. 53-62, August 2002.

[4] E.S. Schubert, D. Hurlbut, P. Adib, S. Oren, "The Texas Energy-Only Resource Adequacy Mechanism", Electricity Journal, vol. 19, no. 10, pp. 39-49, December 2006.

[5] C. Vazquez, M. Rivier, and I. Perez-Arriaga, "A market approach to longterm security of supply," IEEE Trans. Power Systems, vol. 17, no. 2, pp. 349-357, May 2002.
[6] S. S. Oren, "Generation adequacy via call options obligations: safe passage to the promised land," Electricity Journal, vol. 18, no. 9, pp. 2842, November 2005.

[7] W. W. Hogan, On an "Energy Only" Electricity Market Design for Resource Adequacy. Cambridge, MA: Harvard Univ., Sep. 23, 2005

[8] D. Kahneman, A. Tversky, "Prospect Theory: An Analysis of Decision Making under Risk", Econometrica, vol. 47, no. 2, pp. 263-291, March 1979.

[9] J. von Neumann, O. Morgenstern, Theory of Games and Economic Behavior, 2nd edition, Princeton University Press, Princeton, New Jersey, 1947.

[10] D. Feng, D. Gan, J. Zhong, Y. Ni, "Supplier asset allocation in a poolbased electricity market", IEEE Trans. Power Systems, vol. 22, no. 3, pp. 1129-1138, August 2007.

\section{BIOGRAPHIES}

Donghan Feng has been with the faculty of Shanghai Jiaotong University since 2008. He worked at University of Hong Kong (HKU) as a visiting scholar during 2006-2007 and worked at Tsinghua University as a graduate research assistant during 2005-2006. Donghan received his B.Sc. and Ph.D. degree in the department of electrical engineering from Department of Electrical Engineering, Zhejiang University in 2003 and 2008, respectively. Since 2009, he has been granted the Hans Christened Ørsted Award for research with the Centre for Electric Technology, Technical University of Denmark. His research interests are engineering, economic and environmental issues in deregulated electric power industry.

Zhao Xu (S'00, M'06) received $\mathrm{PhD}$. in electrical engineering from University of Queensland in 2006. From 2006-2008, he was an Assistant Professor with Centre for Electric Technology, Technical University of Denmark. Since 2009, he is an Associate Professor at the same institute. His research interests include demand side, grid integration of wind power, electricity market planning and management, AI applications. 